ing letter from some friends. A more marked attack supervened than either of the previous ones. For the four following days the movements continued, and the strychnine seemed to have lost its controlling powers. I therefore considered it best to suspend its employment, a determination which I have since regretted. I ought to have persevered with it, after witnessing the favourable results which had twice followed its employment. As opium, however, had in many cases done essential service, I no longer hesitated to give it a trial. I commenced by ordering three grains of the sulphate of morphia in two ounces of simple syrup; about half a tea-spoonful to be frequently taken, till tranquillity and sleep should have ensued. On the third day we found that the excitement had increased, and not a moment of rest or sleep had been obtained. Six grains of the sulphate of morphia were now ordered in the two-ounce mixture, and as no improvement was manifested at the third dose, the quantity was increased to ten grains. On the fourth day, the motions were still more alarming. The skin of the elbows and arms is excoriated. Other parts of the body, from being dragged hither and thither on the bed, are much reddened. Meanwhile, the morphia has procured two hours' sleep, during which, however, the movements continued without the slightest improvement. Encouraged, however, by the appearance of the sleep, I increased the narcotic salt to one scruple, yet, in the course of the day, the movements augmented to a fearful extent; the arms much swollen, bleeding, and bruised, and looking as if attacked with phlegmonous erysipelas. She dashes her limbs against the bed-posts with tremendous force; the body suddenly arches backwards, so as to form a convexity in front, exhibiting that form which in tetanus would be denominated "opisthotonos;" but this condition is only transient, and the very reverse, or "emprosthotonos," takes place. The head is incessantly rolled from one side to the other. If anything be given her to drink, she endeavours to seize it, being very thirsty, but she has lost all power of directing it to her mouth, and strikes with her forehead, nose, eyes, or lips against the ressel which is offered to her. Her tongue is bruised and lacerated by frequent bites with her teeth.

A sulphur bath was ordered, with subsequent cold affusion, and the dose of morphia to be increased. It required the strength of four strong individuals to hold her in the bath, and they did it with difficulty.

After the bath, the patient slept for a short time; but immediately afterwards the excitement became worse than ever. The skin, throughout its entire extent, is excoriated and red. The incessant movements, in a single day, completely destroyed a bed-covering of very strong linen. As the convulsive movements continued with the same intensity on the following day, and as life could not long endure such suffering, I changed the opium for antispasmodics. In the evening, a restless sleep commenced, which was followed by great nervous excitement and delirium. 'The patient imagined that she was oppressed by some heavy load, and frequently called out, "I am suffocating!"

A loud râle was now audible over the whole chest; the face was pale; the skin hot; the pulse full, and of moderate frequency. Soon afterwards, she fell into a deep coma, and died the next morning.

Upon examination, there was found great excoriation of the skin, and infiltration of blood into the sub-cutaneous cellular tissue. There were sloughs on the left ankle and right elbow. In the brain, there was a slight congestion of the vessels of the pia mater, with distention of the sinuses. No fluid in the ventricles; the membranes are healthy; and after a minute examination of all parts of the brain, no other morbid appearances call be discovered. Nothing abnormal could be discovered in the spinal cord or its membranes. There is a slight congestion of the posterior portion of the lungs, and a quantity of mucus in the bronchial tubes."

In a case which not loug since fell under my observation, the patient was a little girl, aged seven years, of extreme in tellectual powers for one of such tender years. Her replies to my questions were so remarkably sharp as completely to astonish me. The bowels were inactive; the tongue flabby and pale; she suffered firom slight headach; the forehead was extremely prominent; it was feverishly hot. The treatment in this case consisted in the employment of gentle purgatives, - of sulphate of zinc in small doses, both from its stimulating effect on the mucous membrane, and from its general tonic character-a very useful drug in this disease; of cold water, especially applied to the head, from which, also, blood, in very small quantities, was sometimes subtracted by a leech or two. The patient speedily recovered.
Treatment.-The treatment, therefore, in this complaint, must be directed to the following points:-

1st.-Mental and moral quietude. All attempts to excite the intellect or feelings to be avoided. The extreme excitement about the brain to be subdued by occasional slight local depletion,- by the âpplication of cold water, and by keeping the hair short.

2ndly.-The normal activity of the intestines and stomach to be restored, first, by an emetic of sulphate of zinc, to be followed by the employment of brisk purgatives of castor oil, and oil of turpentine; subsequently, small doses of sulphate or oxide of zinc.

3rdly.-General tonic measures. Amongst these, cold water holds the highest place. It should not, however, be applied so as to give a violent shock to the nervous system; but applied either with a sponge or gentle shower-bath.

4thly.-All measures which would have a tendency to congest the brain are to be avoided. Hence, $\mathrm{I}$ would not advise opium, except in cases where all tendency to congestion of the brain, or its membranes, is clearly absent. Even in these cases, camphor, in grain doses, may be advantageously substituted.

As before observed, cold water must not be employed in the terrifying and violent manner which was evidently its mode of employment in the cases translated from the foreign journal. All its beneficial effects may be obtained by a gentle shower bath; or if this cannot be borne, by sponging, or friction, for five minutes, in a wet sheet. Warm-water pediluvia. must not be neglected, as the state of the feet bears an important relation to the condition of the brain. The exercise should be such as nature prompts, and that only. No "plyysical education" quack should be permitted to rack the feeble muscular fibres; and above all should be observed the golden maxim of the great Latin physician, that the exercise should be "citra fatigationem."

As a general rule, spirits, beer, and wine, from obvious reasons, should be avoided. In one case which I attended, I found the mother gave the patient, a very young child, strong beer, and spirits and water, "to brace the nerves," as she expressed it. This, of course, augments the cerebral excitement to a great extent. Sometimes, however, a little ale may be given with advantage. Strychnine may, when used with great caution, produce beneficial results; but when other means will succeed, we are not justified in the employment of such a powerful poison.

Additional remark.-I would beg to call the attention of your readers to the second case of chorea which I have translated, as to the cause of the two relapses - a fright and an unpleasant letter, both powerfully affecting the feelings. Upon some future occasion I trust to bring before their notice a sketch of a peculiar temperament, the "neurotic" and its great connexion as a predisposing cause of this and other diseases of the nervous system.

Bristol, January, 1846

\section{REPORTS OF CASES OF DISEASE OF THE HEART AND OF ANEURISM.}

By Samuel Fenwrck, Esq., Surgeon, North Shields, Lecturer on Anatomy and Physiology at the Newcastle-upon-Tyne School of Medicine and Surgery.

TCBERCUlous DISEASE OF THE HEART.

CASE 1.-A man, aged 45, suffered from symptoms of typhus fever. When I saw him, nine months afterwards, his appearance was strongly indicative of phthisis. He complained of cough, with copious expectoration, oedema of the legs, and great emaciation, which had affected him ever since his recovery from the fever. The pulse was frequent and feeble, and he had once spit blood. The chest was, from accidental circumstances, but hastily examined. The sound, on percussion, was much duller on the left anterior chest than on the right at its upper part. On the right anterior chest, a dull sound commenced at the fourth rib, and extended downwards to the lower edge of the liver, which was felt projecting below the costal cartilages. Posteriorly, the resonance of both sides pretty equal and natural, excepting that dulness commenced inferiorly on the right side at the same point as on the front. Mucous râles existed on both sides, excepting over the dull part of the right chest, where respiration was absent. The heart's sounds were not noticed, nor its extent ascertained by percussion. He sauk a few days after the examination.

Diagnosis.-From the general svmptoms, it was concluded that the patient was affected with phthisis, and this view was 
apparently confirmed by the comparative dulness on the upper and anterior part of the left chest, accompanied by mucous râles.

A utopsy. - The left lung was firmly tied to the chest by adhesions, requiring considerable force to separate them. Anteriorly, at the part where the dulness on percussion had been observed, there was a mass of false membrane, about three lines in thickness, and at various parts throughout the substance of this membrane were oval patches of tuberculous matter. The right lung was also adherent as low as the fourth rib, below which, the cavity of the pleura was filled with serum containing flocculi of lymph. This lung had no covering of false membrane. The substance of both lings was healthy, excepting a few scattered tubercles, mostly hard, and of old formation. The heart was covered anteriorly by a layer of false membrane, of the same description as that which existed upon the left lung. The cavity of the pericardium was completely obliterated. Tuberculous matter was deposited in large masses beneath the lining membrane of the ventricles, and throughout the thickened pericardium, but none was apparent amongst the muscular structure, although it in many parts encroached on the walls of the ventricles. Valves all healthy. Liver rather enlarged, and of a nutmeg appearance. Spleen adherent to the surrounding parts, but otherwise healthy. Other viscera healthy.

Remarks.-First. It is probable, either that the cardiac affection was produced by the fever, or that what was termed typhus had been latent pericarditis, and that the patient being of a scrofulous diathesis, the deposition of tuberculous matter had taken place in the false membranes, in the same way as phthisis often follows congestion or inflammation of the lungs in such persons. I have, however, in other cases, seen deposits of tuberculous matter in the heart, where no other appearance of disease could be detected. 2ndly. The general symptoms were those of phthisis, and when taken in connexion with the local dulness and bronchitis, were quite sufficient to induce the diagnosis of that disease. This case is an exemplification of a class of cases we not unfrequently meet with in scrofulous constitutions, in which gradual thickening of the pleura takes place, with very slight disease of the lungs themselves. Bronchitis is almost always present along with the general symptoms of phthisis, and were it not for the friction sound in an early stage, and the usual position of the dulness in the posterior and inferior part of the chest, it would be exceedingly difficult, if not impossible, to diagnose it from tubercular deposition in the lung.

Carditis AND ENDOCARDITIS.

CASE 2.-A man, aged 30 , of sanguine temperament, had been ill for two or three weeks, and at the time I saw him was labouring under great dyspncea, cough, copious frothy expectoration, general oedema of the body, and fever. There was great anxiety in the expression of his face, the pulse was extremely weak, irregular, and intermittent, and he was frequently attacked with alarming syncope. He had been affected with rheumatic fever six years before, and at that time had suffered from violent pain of the side and other symptoms, apparently arising from pericarditis. Since that period he had been subject to palpitation of the heart, dyspncea on exertion, œdema of the legs, \&c. Percussion over the heart gave a very extensive degree of dulness, but it extended chiefly towards the sternum, and not high up, as in hydropericardium. No undulatory impulse. The heart had a violent struggling action, with occasional intermittence; the sounds very obscure, and unattended with any abnormal murmurs. No dulness on percussion over the lungs, but mucous râles universal. He died a week after the examination, in one of the attacks of syncope, and spit blood during the last two or three days of his life.

Diagnosis.-Fron the struggling action, the absence of fluid, and the general history of the case, adhesion of the pericardium was supposed to exist. The lateral increase of dulness, and the previous symptoms, indicated enlargement of the heart. As no soufflet could be distinguished, it was imagined that no sufficient grounds existed for supposing valvular disease; and in absence of this complaint, the general distress, the state of the pulse, and the obscurity of the sounds, conld only depend upon sottening of the structure of the organ, with the probable formation of polypi in its cavities, both of which were therefore diagnosed.

A utopsy, thirty-six hours after death, (latter end of Norember.)-Body generally odematous, and putrefaction of the skin already commenced. On cutting into the right chest, a quantity of very foetid gas escaped with a whizzing noise. None in the left chest. About a pint and a half of dark bloody fluid existed in the right, and a little clear-coloured serum in the left pleura. Both lungs apoplectic, especially the right; the upper lobes were principally affected, looking like bags of blood. Pericardium seemed as if too small for the heart, which was greatly increased in size. Adhesions existed at its upper part, and also in different points of its lower surface. The adhesions, however, seemed old formations. Heart exceedingly soft; left ventricle vastly dilated and hypertrophied; mitral valve thickened, of a dark-red colour, and looked like a tumid ring; chordae tendina knotted and thickened; adherent polypi in the cavity of the ventricle; aortic valves thickened, and covered with lymph; left auricle of enormous size, but not much hypertrophied; right auricle in the same condition; right ventricle dilated, and the tricuspid valve affected in the same manner as the mitral, but in a less degree. Pulmonary valves soft, but otherwise healthy. Liver congested and soft. Kidneys break down into a pulp, as though they had been macerated for a week.

Remarks.-This case presents two principal points for investigation-1st. Did the softening of the heart exist during life, or was it the result of decomposition? The presence of fotid gas in the pleura and the state of the skin seem to support the latter, but if the hæmoptysis, the continued struggle, frequent attacks of syncope, and peculiar state of the pulse, be considered, the former view will seem most probable. 2ndly. Why did no murmur exist with such a diseased condition of the valves? This may be explained by the exceedingly weak and imperfect contractions of the heart impelling the blood either in very sinall quantities or with a very slight degree of velocity. The absence of a murmur, however, is not dependent upon these conditions alone, as, in other instances, I have heard a bruit alter its character, intermit, or be absent altogether, even when the heart acted freely, and the valves were considerably diseased. At the post-mortem examination, and consequently after the diagnosis was written, $\mathrm{Mr}$. Owen, to whose kindness I was indelted for the opportunity of seeing this patient, stated that he had ascertained the existence of a murmur previous to my examination. As a contrast to this case $I$ have selected the following :-

\section{DISEASE OF THE AORTA.}

CASE 3.-A seaman, aged forty-four, had been for some years subject to violent pains of the shoulder, vomiting, and symptoms of dyspepsia. When he came under my care, he was affected with ascites, and odema of the lower limbs and scrotum. The cedema first commenced, but had been removed by diuretics; the ascites remained, and after the lapse of a month or two the anasarca re-appeared; skin and conjunctivæ yellow; violent pains of the abdomen, and other symptoms of diseased liver; the extent of dulness in the heart's region was greater than usual; the action irregular and intermitting; first sound loud and ringing at the apex, and obscured at the third rib by a loud rasping murmur, which decreased downwards; it sounded very near to the ear all along the upper part of the sternum; second sound loud and clear; pulse full, but rather jerking.

Diagnosis.--Pericardium natural; no effusion; heart enlarged; left ventricle hypertrophied and dilated; structure natural in consistency; mitral valve perhaps a little thiclened, but able to perform its functions; aortic valves thickened, but close perfectly; aorta possibly dilated; it will be found extensively incrusted with bony matter; right ventricles and auricles enlarged.

A utopsy, forty-four hours after death.-Heart enlarged; structure rather flabby; pericardium natural; left ventricle affected with dilatation and hypertrophy; mitral valve slightly thickened, but closesperfectly; columnic carnex hypertrophied; aortic valves quite healthy; aorta healthy, excepting two small depositions of ossific matter at its origin, the larger not more in diameter than a fompenny-piece; both auricles rather dilated; right ventricle dilated, but both the tricuspid and pulmonary valves healthy; liver cinlarged, knotty externally, and internally of a fibrous texture; other viscera healthy.

Remarks--From the perfect state of the second sound at the third rib, it was pretty evident that the aortic valves were able to perform their function, but the thickening of their texture was erroneously inferred from the greatest intensity of the murmur, with the first sound, being immediately over them. The chief point of interest, however, connected with the case, is the very small amount of disease which was sufficient to cause so loud an abnormal sound, and had not the sumptoms of hepatic disease been exceedingly obvious, it might have led to as much mistake in treatment as it did in diagnosis. 
ANEURISM OF THE LEFT ACRICLE.

CAsE 4.-A maltster, aged forty, had, for a considerable period, suffered from cough and expectoration, when, on lifting a heavy sack of malt upon his shoulder, he experienced a sudden pain of the side, accompanied by faintness and sichness. Nine mouths afterwards, his symptoms were cough, great dyspnœa, general cedema of the body and blueness of the face, but this latter not to any very great extent. The heart's space, on percussion, was found extensive, and irregular in shape, but did not extend so high as in hydro-pericardium. Both sounds of the heart deadened, and the first accompanied, near the apex of the heart, by a bellows murmur, which was only audible over a space the size of a five-shilling piece. As the weakness of the patient increased, the murmur gradually altered its character, so that, shortly before his death, it was noticed as an almost continuous sound, most audible during the ventricular systole.

Diagnosis.-From the sudden attack of faintness during exertion, it was imagined a rupture of some internal part had occurred, and the bruit at the apex of the heart seeming to indicate regurgitation through the mitral valve, disease following rupture of that part was diagnosed. From the extensive dulness on percussion, and the deadness of the sounds, hypertrophy was supposed to exist.

Autopsy.-I was unfortunately prevented from seeing this examination, but learned from Mr. Stephens, under whose care the patient was, at the time of his death, the following particulars. The pericardium was natural; the heart generally enlarged; the valves healthy. Projecting from the side of the left auricle was a tumour, the size of a small orange, which, on being cut into, was found communicating with the cavity of the auricle, and almost completely filled with the fibrinous coagula usually found in arterial aneurisms.

Remarks.-This case is exceedingly interesting in three points of view-1st. From its rarity. Mr. Thurnam, in his collection of seventy-four cases of aneurism of the heart, mentions only one of the sacculated description as having occurred in the auricle. 2ndly. On account of the healthy condition of the mitral valve, it having been hitherto invariably found, that aneurism of the auricle has been connected with a contracted state of that valve, preventing the ready exit of blood from the auricle. 3rdly. On account of the difficulty which would be experienced in the diagnosis of a similar case. The bruit with the first sound had been caused by the flow of blood through the neck of the sac during the diastole of the auricle, but being conducted by the parietes of the heart, was most audible at its apex. As the sac, after death, was found almost filled with coagula, the continnous sound was probably produced by the friction of the particles of the blood against the fibrinous clots during both the contraction and dilatation of the auricle. None of the general symptoms would be sufficient to afford us any guide in detecting a future instance of the disease, as they are merely those which usually arise from obstructed circulation. Although a soufflet is not unfrequently found localized near the apex in mitral regurgitation, yet its gradual change into an almost continuous sound of a different character might lead us to suspect some more rare affection of the heart. If, in addition, we should discover irregular enlargement on percussion, and the sudden faintness during or after violent exertion, aneurism of the auricle might be presumed to exist.

\section{ANEURISM OF THE THORACIC AORTA.}

CASm 5.-A bricklayer, aged forty-two, was attacked with pains and a sensation of weight upon ench shoulder. These symptoms continued for some months, when a slight tumour showed itself on the right side of the sternum, near the second or third costal cartilage. I first saw him sixteen months after the commencement of his illness. A tumour, the size of an orange, existed over, and to the right of, the stemun, whilst aroind it there was extensive dulness on percussion. The swelling was elastic, and pulsated with enormous force; no bruit either with the sounds of the heart or over the tumour; first sound of heart deader than usual. The tumour gradually increased in sire, mut the costal cartilages became absorbed, when, not being confined anteriorly, it very rapidly enlarged the reins over it were distunied, and a week or two previous to his death the surfice of the tumour became intlamed, and afterwards blackened to the extent of three inches in diameter. Towards the ciose of lite, the respiration was excedingly laborious, and on examination, a crepitation was heard in the lower and posterior part of the right lung. The power of rision was gradually lost, and he died in a state of coma.
Diagnosis.- Hypertrophy of the heart; aneurism of the aorta at its origin, and softening of the right lung, from compression by the tumour.

A utopsy.-The tumour was formed by an aneurism arising from the origin of the aorta, and projecting through an open$\mathrm{ing}$ in the right side of the stemum. It was filled with fibrine, deposited in layers, and contained, also, a large quantity of coagulated blood. The left ventricle was thickened; no valvular disease. Both lungs greatly engorged and softened, more especially the right, which was compressed by the aneurism.

Remarks.- When a thoracic aneurism appears externally, its heaving impulse is sufficient to explain its nature; where, however, as in this case, much coagulum exists, a bruit is usually absent. It is well worthy of remark, that the first, and, for some time, the only symptoms complained of, were pains about the shoulders. Sympathetic pains are frequently too lightly treated, and $I$ have seen very serious mistakes in diagnosis arise from so doing. I have known the pains of the leg produced by a popliteal aneurism treated for many months as rheumatism, until the size of the tumour in the ham accidentally attracted attention. The character of such pains is, however, totally different from rheumatism, being usually remitting and very violent during the time they are present. Some years ago a patient came under my notice, who complained solely of violent shooting pains in the shoulders and neck. They had resisted all the treatment employed for his relief. On examination, a small tumour was found at the right sterno-clavicular articulation, which proved to be an aneurism. From these and similar cases which could be brought forward, we may lay it down as a practical rule, that wherever violent lancinating or shooting pains exist about the shoulders, the condition of the heart and great bloodvessels should be carefully examined.

\section{ANEURISM OF THE ABDOMINAL AORTA, SIMULATING CALCULUS} IN THE BLADDER.

CASE 6.-A person, aged thirty-two, was admitted into the Newcastle-upon-Tyne Infirmary, in September, 1838, under the care of $\mathrm{Mr}$. Greenhow. He complained of violent pain at the glans penis and pubic region during and after micturition, also of pain in the region of the kidneys, and at the scrobiculus cordis. Urine red, depositing a thick sediment; tongue white, and appetite bad. His illness had been of two years? duration, and was attributed to distress of mind. The symptoms appearing to indicate the presence of a stone in the bladder, a sound was introduced, but no calculus could be detected. Five days after his admission, he was seized with sudden faintness, the countenauce became anxious, the voice whispering, the pulse exceedingly feeble, and the stomach rejected all food. On attempting to rise from his bed, three days afterwards, he was again attacked with sudden fainting, from which he never rallied, but died in a few hours.

Autopsy, thirty-six hours after death. The abdomen was found filled with blood, which had proceeded from the bursting of an aneurism of the aorta. The tumour was the size of a large orange, and had produced caries of the bodies of the vertebra, on which it rested, and to which it firmly adhered. The heart was enlarged; the left ventricle hypertrophied, and the tricuspid valve thickened. No calculus in the bladder, and no appearance of disease in any of the urinary organs.

Remarics.-1st. This case presents an instance of a fact frequently observed in the practice of medicine, that an irritation applied to a nerve is often only indicated by pain in a distant part. Here the pressure upon the aortic plexus produced the usual symptoms of calculus in the bladder, by means, we may suppose, of the communications of the aortic with the hypogastivic plexus of nerves. 2ndly. It would appear, from the sudden depression observed five days after his admission, that the aneurism had burst at that time, but that the hamorrhage had been arrested by natural means until three days afterwards, when the fatal rupture took place.

\section{ABDONINAL AYEURISM.}

CAse 7.-A keelman, aged forty-two, of sanguino-nervous temperament, was admitted into the Newcastle-upon-Tyne Infirmary, under the care of 1 r. Bates. He complained of riolent pain, occuring usually in the moruing, and extending from the left loin to the groin and thich. The pain cansed him to bend forwards on the thigh, and was not increased by pressure on the loin; retraction of the left testicle during the continuance of the attack; pain on passing wrine, which contained no albumen, and was otherwise healthy. The srmptoms gradually increased in violence, the pain extending 
down to the left leg, and the paroxysms taking place three or four times a day, and relieved only by opiates. One morning, when suffering from his usual attack, he was observed by the other patients in the ward to be fainting, and expired a few minutes afterwards.

Autopsy, thirty hours after death-On laying open the abdomen, an immense collection of blood was found behind the peritonæum, extending downwards into the pelvis. It had displaced the intestines, and pushed forward the left kidney. On removing the blood, an aneurism of the aorta was discovered about an inch below the coeliac axis. It had burst on the left side, at the back part, by a ragged hole. The sac, which was about six inches long by four broad, had, by its pressure, caused absorption of the greater part of the bodies of the two last dorsal and two first lumbar vertebra, as well as the heads of the two last ribs. It appeared to be a true aneurism, and contained no coagula. The remains of the bones just mentioned formed a part of its posterior wall, and the blood contained in it must have been in contact with the two last dorsal and two first lumbar nerves of the left side. No calculus existed in the kidney nor ureters, and the viscera were generally healthy.

Remarks.-In this, as in the preceding case, pain was experienced in micturition, probably from the irritation of the sympathetic; but from the greater absorption of the bones, in the present instance, the spinal nerves had also been pressed upon, giving rise to violent pains in their extremities. The nerves, thus exposed, being those nsually affected in calculus of the kidney, the symptoms were well calculated to give rise to the supposition of the existence of that disease. The absence of albumen in the urine being ascertained, the abdomen was examined, and it is difficult to explain how an aneurism of this size could escape detection. From the detail of the last three cases, we find that there are no general symptoms characteristic of aortic aneurism, but that, depending upon pressure on the neighbouring parts, they vary with the situation of the disease. As, however, there is no species of dan. gerous disease so frequently overlooked, we should lay it down as a practical rule, always to examine the aorta by the stethoscope, whenever the symptoms indicate an affection of the contiguous organs, the nature of which may happen to be obscure.

Dockinray-square, North Shields, November, 1845.

\section{REPORT OF A CASE OF CHYLOUS URINE*}

SUCCESSFTLLY TREATED WITH THE BARK OF RHIZOPHORA RACEMOSA, OR MANGROVR, A TREE INDIGENOUS TO BRITXSH GUIANA.

By Georae Reginald Bonxun, M.D., M.R.C.S.L., Georgetown, British Guiana.

THE species of mangrove which I an desirous of bringing to the notice of European practitioners, grows commonly on the sea-coasts, the banks of the rivers as far as the tide extends, and the sides of the salt-water canals of this province. It attains the height of from twenty to thirty feet, with about six or eight inches diameter of trunk; the branches are rather diffuse with terminal tufts of pale-green, rather fleshy leaves, and axillary panicles of insignificant white flowers; the bark is smooth and reddish when fresh cut.

Several species of this genus-as the rhizophora mangel and rhizophora candel-are known to possess astringent properties, being used in some places for tanning. The bark of the rhizophora racemosa lias been long known and appreciated by the negroes, in some parts of this province, as a sovereign remedy in inordinate discharges of urine, but has not, to iny knowledge, been hitherto brought to the notice of the profession.

Before detailing the case of paruria incocta, I may remark, that the disease is by no means uncommon in the West Indies, is very frequently fatal, and as difficult of treatment as the diabetes mellitus, of which it is evidently a modification.

CASE.-II. I _ , aged thirty-one, creole of Demerara, good constitution, bilious temperament, has enjoved good health for several years; on the 8 th of May observed that his urine was turbid, and of a brownish colour; this turbidity increased from day to day, until the urine acquired a milky appearance, and was slightly increased in quantity; he gradually lost his strength and appetite. Took tincture of iron drops three times a day.

* Pararia incocta of Mason Good-Study of Medicine, vol. v., third edition, p. 512 .
June 8th.-Urine decreased in quantity, quite white, and coagulating immediately after being passed, so as to resemble blancmange; dreadfully weak; little appetite; costiveness; dry skin; emaciation; low spirits; no perspiration at any time during the day. Took balsam of copaiba, liquor of potass, Scio turpentine, and other medicines, without any relief.

July.-Symptoms the same; much emaciated. Took indigo in ten-grain doses, and restricted himself to meat diet, with milk for drink. Temporary relief for a short time, when all symptoms returned. 'Took Dover's powders in ten-grain doses, and applied a blister to the spine.

August.- Kept reclining posture, persisted in meat diet, and took wine of iron. At this period felt great thirst.

5th month.-Took calomel and opium to salivation; then infusion of bark, with rhubarb and cinnamon, and then soda and ammoniacal gum, without experiencing the slightest benefit, the urine continuing to coagulate firmly, and a considerable quantity of mucus being likewise discharged from the urethra. About the end of the fifth month, took compound rhubarb pill and ipecacuanha, when an amelioration of all the symptoms took place; the urine became limpid and natural in quality; there was no thirst or inclination to drink, and a very trifling perspiration. In three days all the symptoms returned. He continued the rhubarb and ipecacuanha and took, at the same time, quinine and iron, but experienced no further relief.

In the sixth month, abandoned the meat diet, but ate very little, and took for his drink a very small quantity of milk-andwater. Took infusion of angostura, with carbonate of iron, and likewise citrate of iron and quinine. General health somewhat better, urine remaining the same.

The seventh month: very much emaciated; general health miserable; cramps during the night; not the slightest perspiration; a loathing of fluids. Tried infusion of bucha with soda, hydriodate of potass and various indigenous herbs, without relief.

At the end of the ninth month, took benzoic acid, increasing the dose from ten to twenty grains three times a day; general health rather better during a week, and then became as bad as ever. Also took Pareira brava with no benefit.

Tenth month.-Went into the country; no medicine nor particular diet, excepting an abstinence from strong drink. After being in the country three weeks, was in the following state: urine, three pints daily, passed at four or five times, mostly in the evening, white, firmly coagulating, and separating, after remaining some hours, in to a substance resembling curds and whey; no odour nor saceharine taste; the mucous discharge, which was hitherto only produced by exertion during the day, now came on an hour after rising in the morning, with great prostration of strength; tongue furred and dry; eyes suffused; lips parched; excessive languor and debility slight perspiration occasionally; more inclination to drink, and appetite rather better; violent cramps during the night; greatly emaciated, but still able to move about a little. Took decoction of cashew bark, (anacardium occiclentale, ) and thought himself slightly relieved for a time, but the symptoms returned and continued with violence until the middle of March, the eleventh month, when, by the advice of an old negress, he took decoction of rhizophora bark, in ounce doses, four times a day. After two days, urine appeared, immediately after being passed, like ill-prepared camphor mixture, and coagulated imperfectly; general health rather better. In five days, (the same treatment being continued,) urine not coagulating, more limpid, with flowing particles of mucus; perspiration gradually increasing; appetite better and more natural; felt generally much improved. In seven days, felt so well that he discontinued medicine for two days, when symptoms began to retum, urine to coagulate, \&c. Medicine was resumed in increased quantity, and continued for several days, until all the symptoms entirely disappeared, and the patient was restored to perfect health. After remaining in good health for some time, Mr. K- was again attaclied, and had again recourse to the mangrove bark, from which he experienced immediate relief, but being obliged to visit the town, and nor having a sufficient supply, the distase increased upon him considerably, but was arrested as suon as he procured the medicine.

Within the last few months, this gentleman, in whom there appears to be a strong terdency to this peculiar disesse, has had another attack in a modified form, the nine being similar to fresh milk in odour, tiste, and appearance, and separating by anplication of wine into curds and whey. The nse of the baris restored him immediately. It mar be observed, that although he lins liad tro slight returns of the disease, it has 